\title{
Transdermal Drug Delivery System-Quality by Design Approach
}

\section{Alok Bandyopadhyay*}

RAC, AB Consulting, Lansdale, Pennsylvania 19446, USA

\begin{abstract}
Transdermal Drug Delivery System (TDDS) is a delivery system of continuous drug penetration through the skin at a reproducible rate over the intended application time period. This process is accomplished by two technologies: (a) Passive, and (b) Active. The former uses particulate system or chemical penetration system enhancers, whereas active technology uses electrical device to overcome skin barriers. Some of the major reported products have quality issues. Several guidance's are published on this aspect. For better understanding, FDA suggested to perform the development of delivery system through Quality by Design (QbD) approach.

Although there are some limitations e.g. skin tolerability, skin barrier properties and variability of application site conditions, but also there are several advantages. Convenience, visibility of drug administration, consistent serum levels through steady permeation of drug across the skin, suited for delivery of drugs unstable for GI tract, removal of patch due to adverse event are all in the advantage category. So it is suggested that all critical parameters should be finalized in the pharmaceutical manufacturing development phases. The Quality Target Product Profile (QTTP) is required to establish a consistent quality product devoid of any quality issues. Overall, essential parameters are to be finalized in pharmaceutical development in order to devoid of any quality issues.
\end{abstract}

Keywords: Transdermal Drug Delivery System (TDDS); Skin barriers; Pharmaceutical

\section{Introduction}

The skin is the largest organ of the body and it protects the body from the environment al factors. In order to achieve therapeutic values of a drug product delivered through skin, it is required to overcome the skin barriers. This is accomplished generally by using two technologies: (a) Passive, and (b) active. Passive technologies use particulate system like liposome; transfer some, micro emulsions, or solid lipid nanoparticles. Sometimes, passive technologies use chemical penetration enhancers like glycols or terpenes in the formulation. On the contrary, active technologies overcome skin barriers through microneedles, or low intensity ultrasound, magnetospheres, electroporation (short, high voltage pulse) or iontophoresis (weak continuous electric current). A transdermal patch, which may also be considered a Transdermal Drug Delivery System (TDDS), is defined as a flexible, multi-layered, pharmaceutical single dose preparation of varying size containing one or more active substances to be applied to the intact skin for systemic absorption. This is normally formulated with pressure-sensitive adhesives that assure the adhesion of the preparation to the skin. A transdermal patch includes a backing sheet, impermeable to the active substance and normally impermeable to water. The releasing surface of the patch is covered by a protective liner to be removed before applying the patch to the skin. The rate limiting step for systemic absorption of the active substance is either usually through the skin or dissolving the active substance in a (semi solid) reservoir, with a membrane to control the release and the diffusion of the active substance(s) from the patch [1-3].

Several transdermal patches dominate market since early part of 2000. Some of them are smoking cessation patch, some hormonal deficiency patch. Gelnique (oxybutynin) came into market March 2009 to treat the patients of overactive bladder. In May 2011 US approved microneedle based influenza vaccine patch. Single use battery powered iontophoretic sumatriptin patch was approved in 2012. Neupro (rotigotine) patch became available in market for Parkinson disease patients in early part of 2013 [4].

Some of the major reported products have quality issues. Mainly, the patches did not stick to the skin and it easily came off. Sometimes the patches leaked or wrinkled. It was also noted that the liner became difficult to remove from the patch and sometimes active substance sticks inside the pouch. Fentanyl reservoir patches were recalled as Class I in 2004. Safety concerns and some efficacy issues were reported in hormonal contraceptive in 2005. Issues of poor skin adhesion and cold flow were seen in ADHD patch in 2007. Snowflakes (Dry crystallization) were found in Parkinson's disease patch in 2008 [5]. In order to better understand FDA suggested performing the development of delivery system through Quality by design (QbD) approach. The purpose of this article is to discuss the QbD approach in the development of TDDS which may help to generate quality products.

\section{Road map for the product}

The road map of the product is shown in Table 1.

\section{Pharmaceutical Development}

All ingredients of the TDDS have to be well tolerated by the skin and support continuous drug penetration through the skin at a reproducible rate over the intended application time period. All issues have to be taken care of during pharmaceutical development which is stated in the following categories:

\section{Selection of drug product}

A formulation development starts after the selection of drug substances and excipients. The Physical properties of formulated drug product, characteristics of polymorphs and control of such polymorphs are considered to be studied in details. Moreover, the formulation of

*Corresponding author: Alok Bandyopadhyay, RAC, AB Consulting, Lansdale, Pennsylvania 19446, USA, E-mail: akbandy@gmail.com

Received July 18, 2017; Accepted August 22, 2017; Published August 29, 2017

Citation: Bandyopadhyay A (2017) Transdermal Drug Delivery System-Quality by Design Approach. J Bioanal Biomed 9: 217-219. doi:10.4172/1948-593X.1000181

Copyright: @ 2017 Bandyopadhyay A. This is an open-access article distributed under the terms of the Creative Commons Attribution License, which permits unrestricted use, distribution, and reproduction in any medium, provided the original author and source are credited. 


\begin{tabular}{|l|l|}
\hline 1. & Drug selection and polymorph control \\
\hline 2. & Adhesive selection \\
\hline 3. & Assessing the penetration of enhancer \\
\hline 4. & Crystal seeding studies \\
\hline 5. & Selection of patch design \\
\hline 6. & $\begin{array}{l}\text { Stability assessment for: } \quad \text { (a) Compatibility, and (b) Cold flow and } \\
\text { crystallization }\end{array}$ \\
\hline 7. & $\begin{array}{l}\text { Adhesion test battery to measure and tape properties } \\
\text { Flux studies using diffusion cells and preclinical animal studies }\end{array}$ \\
\hline 8. & Flux studies using diffusion cells and preclinical animal studies \\
\hline 9. & $\begin{array}{l}\text { Wear studies as part of Bioequivalence (BE) to establish study arms to } \\
\text { assess adhesion, cold flow, and effect of overlays }\end{array}$ \\
\hline
\end{tabular}

Table 1: Road map of the product.

passive systems has to promote drug partitioning into and transport across the skin layers, to maintain drug activity gradient across the skin nearly constant over the intended application time and to assure a high drug depletion rate of the patch.

\section{Characteristics of permeation enhancers}

The stratum carenum is a formidable barrier to exogenous agents including drugs. Therefore, it is often necessary to add permeationenhancing chemicals to aid drugs in passing through the stratum carenum. Recognizing the need to increase skin permeability, delivery strategies have turned largely to the development of chemical enhancers

\section{Selection of adhesive and solubilization properties}

Solubility assessment is also another important critical factor for adhesive, additive, penetration enhancer solvent, plasticizer, solvent of investigative formulation. The adhesives are exposed to different drug concentration. Stability of patches is determined at room temperature and elevated temperature $\left(50^{\circ} \mathrm{C}\right)$. Identification test is performed to monitor drug crystal growth over a few weeks. Crystal seeding studies are performed by preparing drug adhesive mixture with various drug concentrations and by seeding drug crystals of all polymorphs onto the surface. Observed growth or dissolution of seeded drug crystals can be predictors of pot crystallization during storage.

Adhesion selection and control of cross-linking Resin, polymer component and functional groups, molecular weight, resin to polymer ratio. Addition of cohesive strengthening agents: non-ionic surfactants, fatty acid esters of glycerol, metallic salts of fatty acid and phosphoric acid. For transdermal patches an optimum balance between adhesion and cold flow properties of the adhesive formulation has to be adjusted to prevent detachment of the drug release surface from the skin surface during application or cohesion failure of the adhesive which might lead, for example, to drug containing adhesive residues on skin or clothes. Accordingly, state-of-the-art optimization of adhesion properties is an essential part of TDDS formulation development and an integral part of general regulatory requirements on quality, as summarized below:

Properties of tack filers or viscosity inducing agent and antioxidant property: Another critical attributes is rheological properties which consists of viscous modulus, elastic modulus, intrinsic viscosity, glass transition temperature. In addition, molecular weight distribution, permeability, compatibility, antioxidant property, stability and leachable are all important parameters.

Liners backing layers and rate limiting membrane: Liners, backing layers and rate controlling membranes are other important items to address. Physical dimension, degree of porosity, pore dimension for acceptance quality limit, tensile modulus, elongation modulus, moisture, water, and oxygen transmission rate is all part of these items.

Roll force, line speed, substrate tension, registration and lamination pressure are the parameters for process operation. There are several quality risks to be considered e.g. fold over defects, incorrect seal thickness, leakage, and evaporation are the quality risks.

Tape properties consist of wettability (measured through tack), Shear/creep resistance (measured through resistance to flow), and adhesion strength (measured by a force required to peel). are all critical quality attributes?

\section{Design and development}

After the development of initial parameters patch design and process design are the next steps, which are required to be verified through scale up process. Formulation Optimization is performed by Design by experiment. Effect of temperature and occlusion, impact on drug flux through stability of thermodynamic changes, crystallization, polymer crosslinking etc. are the subjects of study. To claim bio waivers IVIVR/IVIVC is required to establish. Manufacturing changes and critical process controls are listed in Table 2.

\section{Quality Target Product Profile (QTPP)}

Target Product Profile (TPP) is planning tool for the development of candidate from discovery to clinical development programs. This establishes safety, efficacy, and marketing suitability of the drug. Quality target product profile(QTTP), dealing with the quality factors, is also a part of TTP Table 3 shows various parameters of QTTP, which are required to examine for the safety of the product.

\section{Advantage and Limitation}

Basic assessment criteria for transdermal drug administration have to consider a number of benefits, and drawbacks, as listed below.

\section{Advantages}

There are several advantages of TDDS, which are described in the following: (1) Convenience, Visibility of drug administration and patient compliance, (2) Steady permeation of drugs across skin assures

\begin{tabular}{|c|c|}
\hline Processing steps & $\begin{array}{l}\text { a. Raw materials controls } \\
\text { b. Adhesive mixing operations } \\
\text { c. Adhesive coating and drying } \\
\text { d. Gel dispensing (Reservoir) } \\
\text { e. Patch sealing process } \\
\text { f. Primary packaging }\end{array}$ \\
\hline Process controls & $\begin{array}{l}\text { g. Controls to minimize air entrapment to drug adhesive or } \\
\text { drug polymer mixture } \\
\text { h. Controls to maintain drug homogeneity and viscosity of } \\
\text { drug polymer mixture in gel pots } \\
\text { i. Engineering controls to guide webs during lamination to } \\
\text { prevent fold-over defects } \\
\text { j. Establishing sensors for continuous monitoring critical } \\
\text { operations and weeding out defective patches e.g. } \\
\text { patch registration, heat-seal thickness, drug in heat- } \\
\text { seal area } \\
\text { k. All line testing for pouch seal integrity: } 100 \% \text { inspection } \\
\text { by vision }\end{array}$ \\
\hline $\begin{array}{l}\text { Critical process } \\
\text { controls }\end{array}$ & $\begin{array}{l}\text { I. Laminator speed: range and target } \\
\text { m. Laminator roll pressure: range and target } \\
\text { n. Payou\#1 tension range and target } \\
\text { o. Payout\#2 tension range and target } \\
\text { p. Upper rewind tension range and target } \\
\text { q. Laminator roll size limits }\end{array}$ \\
\hline
\end{tabular}


1. Deliver the required dose. Minimize variability of drug absorption

2. Maintain adequate physical stability and physical integrity of the TDDS during storage and application

3. Minimize the excess amount of drug remaining in the TDDS after application

4. Assure good adhesion and cohesion properties

Adhesion to skin for about 1-7 days without additional overlays. Minimize

5. risk of detachment during application. Minimize variability of skin adhesion and cold flow

6. Easy removal from skin with no trace of adhesive on skin

7. Maintains required flux throughout the wear period and Use of overlays does not change in flux

8. No or minimize skin irritation and allergic reaction (minimize)

9. Works under physically active lifestyle. Moisture resistance (skin wear ability, sweating, showers, sauna etc.)

Table 3: Quality target product profile.

consistent serum levels, (3) Lack of peaks and troughs, (4) Avoidance of first pass metabolism, (5) Suited for delivery of drugs unstable in GI tract, (6) Ionic drugs delivered using active delivery technologies, (7) Patch may be removed due to adverse event (exception depot effect), and (8) less permeable drugs and large molecular drugs may be delivered by permeation.

\section{Limitations}

There are several limitations which are: (1) Skin tolerability, (2) Skin barrier properties, and (3) Variability of application site conditions.

\section{Regulatory Guidance}

The systems' design can range from drug-in-adhesive matrix systems to more complex systems that require microelectronics. Several guidance's were provided related to the development of issues of TDDS [1-3,6-10]. Transdermal products have properties that may lead to skin irritation and/or sensitization. The delivery system, or the system in conjunction with the drug substance, may be responsible for these reactions. To minimize these adverse events, guidance provided recommendations required to perform during drug development [1].

Existing TDDS and topical patches contain a larger amount of the drug substance than what is intended to be delivered to the patient. This excess amount of drug substance is needed to facilitate delivery of the intended amount of the drug to the patient and remains as residual drug in the used system. The amount of residual drug substance in TDDS and topical patches has a significant potential to impact the products' quality, efficacy, and safety (including abuse potential). Consequently, it is necessary to ensure that an appropriate scientific approach is used to design and develop these products. The approach should ensure that the amount of the residual drug substance is minimized consistent with the current state of technology [1-3].

A prolonged release dosage form may be acceptable if the active substance can produce the desirable clinical effect with a different PK profile than that resulting from an immediate-release form. A prolonged release formulation may offer several advantages over an immediaterelease form 8 . This guidance provides recommendations for the design and conduct of studies evaluating the adhesive performance of a TDDS or a topical patch submitted in support of an Abbreviated New Drug Application (ANDA) [2].

A QbD approach can facilitate the development of TDDS and topical patches designed to meet patient requirements and post-use considerations. In particular, it can aid in developing a product to deliver the optimum amount of drug across the skin while minimizing the amount of drug load, thus resulting in the least possible amount of residual drug substance. QbD is applicable to both new products and reformulation of existing products. Moreover, it can lead to a better understanding and continual improvement to the product throughout its lifecycle $[9,10]$.

\section{Further Advancement}

Companies are using both passive and active technologies to improve the delivery system. Within passive technologies Nanotechnologies seem to hold some hope of expanding the transdermal market. NewGen Biopharma and Particle Science have developed a proprietary delivery system using nanotechnology. On the other hand, in active technologies, electrical devices are in development or in the market to provide treatment of specific diseases, including Travanti Medical's Iontopatch (low voltage, continuous), an alternative to injection for treatment of tendinitis and Inovio's electroporation (high voltage, short duration) for vaccine delivery. In addition, Transdermal specialties developed U Strip, an active transdermal delivery system, uses alternating ultrasonic waveforms to enlarge the diameter of the skin's pores enabling large molecule drugs to permeate through the skin into dermis and then into blood stream [10].

\section{Conclusion}

TDDS is a delivery system of continuous drug penetration through the skin at a reproducible rate over the intended application time period. For better understanding, FDA suggested to perform the development of delivery system through QbD approach. Advantages of this approach are discussed earlier.

Target Product Profile (TPP) is planning tool for the development of drug candidate from discovery to clinical development programs. This establishes safety, efficacy, and marketing suitability of the drug product. One of the product profiles QTTP, which deals with the quality factors, is required to finalize in the early part of the program for yielding consistent quality products. In addition, optimization of formulation in Design Development, controls and critical pathways should be finalized during pharmaceutical manufacturing development phase.

\section{References}

1. USFDA (1999) Guidance for industry: skin irritation and sensitization testing of generic transdermal drug products. CDER, Fishers Lane, Rockville, USA.

2. USFDA (2011) Guidance for industry: residual drug in transdermal and related drug delivery systems.

3. USFDA (2016) Assessing adhesion with transdermal delivery systems and topical patches for ANDAs. CDER, New Hampshire Ave, Hillandale Bldg, Silver Spring, USA.

4. https://en.wikipedia.org/wiki/Transdermal_patch

5. Wright $P(2013)$ Transdermal drug delivery looks for new frontiers. Online Edition.

6. EMA (2014) Guideline on the quality of Transdermal Patches: European.

7. EMA (2014) Guideline on the pharmacokinetic and clinical evaluation of modified release dosage forms.

8. USFDA (2017) Pharmaceutical quality/CMC

9. USFDA (2015) Nonclinical safety evaluation of reformulated drug products and products intended for administration by an alternate route: guidance for industry and review staff, good review practice. CDER, New Hampshire Ave, Hillandale BIdg, Silver Spring, USA.

10. http://transdermalspecialties.com/home.html 